\title{
Ascomicetos hipogeos de la región occidental del Sistema Volcánico Transversal, México
}

\section{Hypogeous Ascomycetes of the western portion of the Transversal Volcanic System, Mexico}

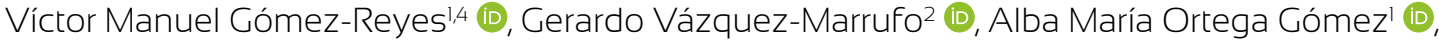 \\ Gonzalo Guevara Guerrero ${ }^{3}$ [D
}

1 Universidad Michoacana de San $\mathrm{Ni}$ colás de Hidalgo, Facultad de Biología, Herbario EBUM, Edificio R planta baja, Ciudad Universitaria, Av. Francisco J. Mújica s/n, 58030 Morelia, Michoacán, México.

2 Universidad Michoacana de San Nicolás de Hidalgo, Facultad de Medicina Veterinaria y Zootecnia, Centro Multidisciplinario de Estudios en Biotecnología, Posta Veterinaria km 9.5 carretera Morelia-Zinapécuaro, 58893 Morelia, Michoacán, México.

3 Instituto Tecnológico de Cd. Victoria, Av. Portes Gil 1301 Pte., 87010 Cd. Victoria, Tamaulipas, México.

4 Autor para la correspondencia: vmgomez@umich.mx

Recibido: 8 de diciembre de 2017 Revisado: 24 de enero de 2018. Aceptado: 6 de marzo de 2018. Primero en línea: 6 de agosto de 2018.

Publicado: 3 de octubre de 2018

Citar como:

Gómez-Reyes, V. M., G. Vázquez-Marrufo, A. M. Ortega Gómez y G. Guevara Guerrero. 2018. Ascomicetos hipogeos de la región occidental del Sistema Volcánico Transversal, México. Acta Botanica Mexicana 125: 37-48. DOl: $10.21829 / \mathrm{abm} 125.2018 .1327$

$\mathrm{DOI}$

$10.21829 / a b m 125.2018 .1327$

\section{RESUMEN:}

Antecedentes y Objetivos: En México se han reportado 42 especies de ascomicetos con hábito hipogeo; son pocas considerando el conocimiento de otros grupos de hongos. El objetivo de este trabajo fue incrementar el conocimiento sobre la diversidad y distribución de especies de hongos hipogeos para México. Métodos: Se realizaron recolectas en diversos tipos de vegetación del Sistema Volcánico Transversal en la porción occidental perteneciente al estado de Michoacán. El material se fotografió y describió siguiendo los protocolos para el grupo.

Resultados clave: Se presentan la descripción y discusión de seis especies de ascomicetos con hábito hipogeo, Elaphomyces appalachiensis, Genea mexicana, Genabea cerebriformis, Hydnobolites cerebriformis, Tuber pseudoseparans y T. tequilanum.

Conclusiones: Los registros representan nuevas localidades para el país y primeras citas para la micobiota del estado de Michoacán. Se incrementa la distribución de Elaphomyces appalachiensis, Genabea cerebriformis e Hydnobolites cerebriformis, conocidos de los Estados Unidos de América y el Norte de México, hasta el Sistema Volcánico Transversal, mientras que Genea mexicana, Tuber pseudoseparans y T. tequilanum solo se conocen en este sistema montañoso.

Palabras clave: diversidad fúngica, Michoacán, micobiota, pseudotrufas, trufas.

\section{ABSTRACT:}

Background and Aims: In Mexico, 42 species of ascomycetes have been reported with hypogeous habit, which are few considering the knowledge of other groups of fungi. The purpose of this paper is to increase the knowledge about the diversion and distribution of hypogeous fungi species for Mexico. Methods: Specimens were collected from different types of vegetation in the western portion of the transverse volcanic system, located in the state of Michoacán. The specimens collected were pictured and described following the protocols for the group.

Key results: We present the description and discussion of six species of ascomycetes with hypogeous habit, Elaphomyces appalachiensis, Genea mexicana, Genabea cerebriformis, Hydnobolites cerebriformis, Tuber pseudoseparans and T. tequilanum.

Conclusions: The records of these species represent new collection sites for Mexico and first records for the mycobiota of the state of Michoacán. It increases the distribution of Elaphomyces appalachiensis, Genabea cerebriformis and Hydnobolites cerebriformis, known from the United States of America and northern Mexico, to the Transversal Volcanic System, while Genea mexicana, Tuber pseudoseparans and T. tequilanum only are known in this mountainous system.

Key words: fungal diversity, Michoacán, mycobiota, pseudotruffles, truffles. 


\section{INTRODUCCIÓN}

En México el conocimiento sobre la diversidad y distribución de especies de hongos hipogeos aún es escaso; la mayoría de los registros pertenecen a Nuevo León y Tamaulipas, en el norte del país. El primer registro de un hongo con hábito hipogeo para México corresponde a Melanogaster variegatus (Vittad.) Tul. \& C. Tul. var. mexicanus Farlow (Lumholtz, 1902), la cual posteriormente fue descrita como la nueva especie Melanogaster umbrinigleba Trappe \& Guzmán (Trappe y Guzmán, 1971) y actualmente presenta una modificación por variante ortográfica a Melanogaster umbriniglebus de acuerdo con el Index Fungorum (2017).

Las especies de hongos hipogeos, también conocidas como trufas o pseudotrufas, se clasifican dentro de los Phyla Glomeromycota, Ascomycota y Basidiomycota, así como en taxa anteriormente asociados a Zygomycota, éste último actualmente subdividido (Hibbett et al., 2007). Con relación a las especies hipogeas de Ascomycota, en México, se han registrado representantes de los géneros Elaphomyces Nees, Carbomyces Gilkey, Delastria Tul. \& C. Tul, Genea Vittad., Geopora Harkn., Gilkeya M.E. Sm., Trappe \& Rizzo, Hydnobolites Tul. \& C. Tul., Hydnotrya Berk. \& Broome, Pachyphlodes Zobel (= Pachyphloeus), Mattirolomyces E. Fisch. y Tuber P. Micheli ex F.H. Wigg., en los cuales se incluyen 42 especies y dos variedades (Trappe y Guzmán, 1971; Trappe et al., 1979; Garza et al., 1985; Cázares et al., 1992; Smith et al., 2006; Trappe y Cázares, 2006; Guevara et al., 2008a; Healy et al., 2009; Kovács et al., 2011; Castellano et al., 2012; Gómez-Reyes et al., 2012; Guevara et al., 2012; Moreno et al., 2012; Guevara et al., 2013; Gómez-Reyes et al., 2017; Piña-Páez et al., 2017).

Estos hongos juegan un papel relevante en el equilibrio dinámico de los ecosistemas forestales, por dos razones principales:

1. Son parte de la dieta de un gran número de especies animales. Cuando las esporas están maduras, los cuerpos fructíferos emiten sustancias orgánicas volátiles (Splivallo et al., 2011), que son detectadas por ardillas, ratones, tlacuaches y venados, entre otros, que consumen los cuerpos fructíferos, dispersando las esporas a través de la defecación (Fogel y Trappe, 1978; Maser et al., 2008; Nuske et al., 2017).

2. La mayoría de las especies de hongos hipogeos forma micorrizas (Ouanphanivanh et al., 2008; Shefferson et al., 2008), asociaciones que se establecen entre el micelio del hongo y las raíces de un gran número de plantas gimnospermas y angiospermas, incluyendo orquídeas, en la que ambos organismos resultan beneficiados.

Para el estado de Michoacán, se tienen registradas 13 especies, de las cuales cinco son Ascomycota: Mattirolomyces spinosus (Harkn.) Kovács, Trappe \& Alsheikh (Kovács et al., 2011), de la Sierra Madre del Sur, Elaphomyces aculeatus Vittad., E. maculatus Vittad., E. muricatus Fr. (Gómez-Reyes et al., 2012) y Delastria rosea Tul. \& C. Tul. (Gómez-Reyes et al., 2017) para el Sistema Volcánico Transversal. Ocho corresponden a Basidiomycota, todas de esta misma región fisiográfica: Rhizopogon michoacanicus Trappe \& Guzmán (Trappe y Guzmán, 1971), R. ochraceorubens A.H. Sm. y R. subcaerulescens A.H. Sm., (Hosford y Trappe, 1980), Hysterangium latisporum Castellano, Cázares \& G. Guevara (Guevara et al., 2008b), Trappea darkeri (Zeller) Castellano (Gómez-Reyes et al., 2014), Stephanospora michoacanensis G. Guevara \& Castellano (Guevara et al., 2015a), Aroramyces balanosporus G. Guevara \& Castellano y A. herrerae G. Guevara, Gomez-Reyes \& Castellano (Guevara et al., 2016).

El objetivo de esta contribución es ampliar el conocimiento de los hongos hipogeos del Sistema Volcánico Transversal del estado de Michoacán y del país.

\section{Materiales y Métodos}

El muestreo se realizó en seis localidades del Sistema Volcánico Transversal (Cuadro 1), en la porción que corresponde al estado de Michoacán. El material recolectado se estudió siguiendo las recomendaciones de Castellano et al. (1989) y Pegler et al. (1993). Los colores de los ejemplares en fresco se basaron en la carta de identificación 
Cuadro 1: Localidades de las especies estudiadas, coordenadas geográficas y tipos de vegetación de acuerdo a Rzedowski (1978), en el estado de Michoacán, México.

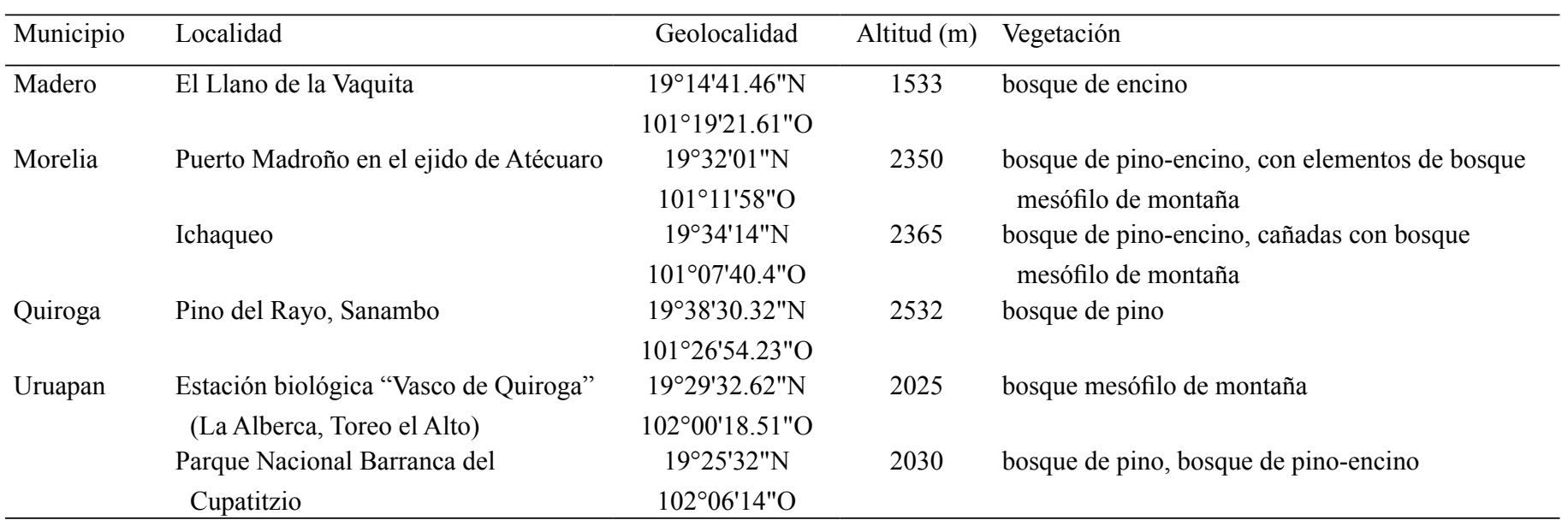

de colores del Royal Botanic Garden Edinburgh (RBGE, 1969). Para examinar características microscópicas (ascas, ascosporas, trama himenial y peridio), se montaron preparaciones temporales de ejemplares deshidratados, realizando cortes a mano y se montaron en $\mathrm{KOH}$ al 5\%, agua y Reactivo de Melzer. Se consideraron 30 medidas de las estructuras microscópicas (objetivo de 100×) con un microscopio (Motic BA300, Kowloon, Hong Kong) con cámara digital para microscopía (Moticam 2300, Kowloon, Hong Kong) y el software Motic Image Plus 2.0 (Motic, 2004). Se estimó el estadístico Q de acuerdo con Largent et al. (1984). Los ejemplares estudiados se depositaron en la Colección de macromicetos del Herbario EBUM de la Facultad de Biología de la Universidad Michoacana de San Nicolás de Hidalgo con un duplicado en el Herbario José Castillo Tovar ITCV del Instituto Tecnológico de Ciudad Victoria.

\section{Resultados}

Elaphomyces appalachiensis Linder, J. Elisha Mitchell Scient. Soc. 55: 133. 1939. Figs. 1A-C.

Ascoma $14 \times 16 \mathrm{~mm}$ de diámetro, globoso a subgloboso, completamente cubierto por micelio color morado (76) a violeta (77); peridio $\pm 1 \mathrm{~mm}$ de grosor, color azul verdoso (67), reacción positiva al $\mathrm{KOH}$ cambiando de color morado (76), violeta (77) a vináceo negruzco (38); gleba polvorienta color azul verdoso oscuro (74) a negruzco (38), con numerosas venas color blanco que se unen al peridio; olor y sabor no registrados; peridio tres capas; epicutis 190-250 $\mu \mathrm{m}$ de grosor, color morado (82) a violeta pálido (79), compuesto de hifas septadas, 3-6 $\mu \mathrm{m}$ de ancho, laxamente entremezcladas, pared delgada; mesocutis $250-350 \mu \mathrm{m}$ de grosor compuesto de hifas septadas, hialinas, pared delgada, numerosas células globosas a isodiamétricas, 8-20 $\mu \mathrm{m}$, las cuales se van haciendo más grandes conforme se acerca al subcutis; subcutis 150-300 $\mu \mathrm{m}$ de grosor, formando una capa parenquimatosa color café pálido a verde claro, compuesta de células isodiamétricas, 8-30 $\mu \mathrm{m}$, pared gruesa de $2 \mu \mathrm{m}$; ascas globosas, 20-28(31) $\mu \mathrm{m}$, hialinas, pared gruesa, octosporadas; ascosporas globosas, 8.5-11.5 $\mu \mathrm{m}$ de diámetro $(\mathrm{Q}=1.0-1.06)$, sin ornamentación, color verde oliva pálido (56) en $\mathrm{KOH}$, color verde amarillento en masa, espinas cortas, 0.8$1.2 \mu \mathrm{m}$ de alto.

Hábito: hipogeo, solitario a gregario bajo Carpinus caroliniana Walt. 

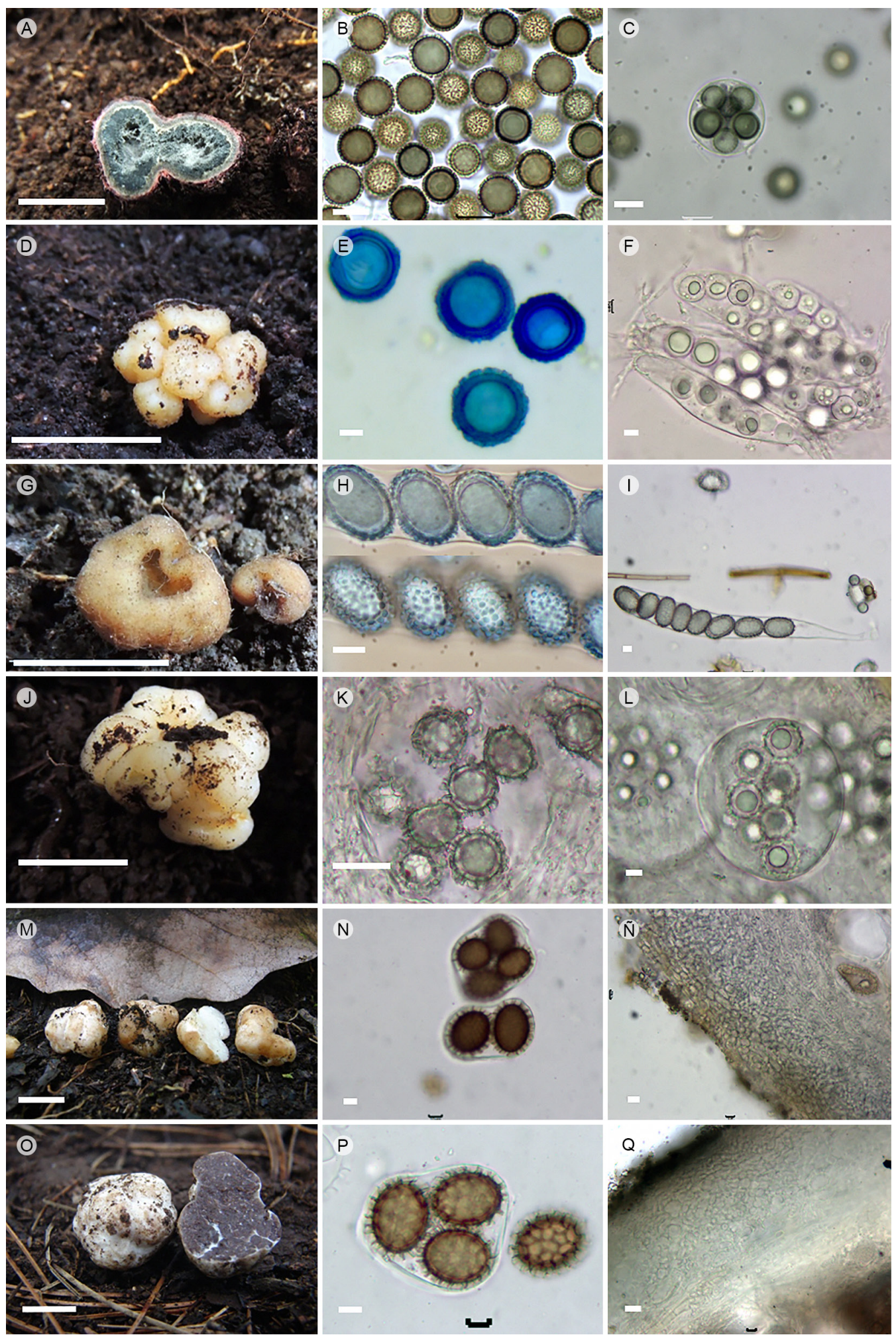

Figura 1: Elaphomyces appalachiensis Linder (Gómez-Reyes 889): A. ascoma (barra=10 mm); B. ascosporas (barra=10 $\mu \mathrm{m})$; C. asca (barra=10 $\mu \mathrm{m})$. Genabea cerebriformis (Harkn.) Trappe (Gómez-Reyes 778): D. ascoma (barra=10 mm); E. ascosporas (barra=10 $\mu \mathrm{m})$; F. asca (barra=10 $\mu \mathrm{m})$. Genea mexicana Guevara, Göker \& Stielow (Gómez-Reyes 852): G. ascoma (barra=10 mm); H. ascospora (barra=10 $\mu \mathrm{m}$ ); I. asca (barra=10 $\mu \mathrm{m})$. Hydnobolites cerebriformis Tul. \& C. Tul. (Gómez-Reyes 876): J. ascoma (barra=10 mm); K. ascosporas (barra=10 $\mu \mathrm{m}$ ); L. ascas (barra=10 $\mu \mathrm{m})$. Tuber pseudoseparans G. Guevara, G. Bonito \& J. Trappe (Gómez-Reyes 874): M. ascoma (barra=10 mm); N. ascosporas y ascas (barra=10 $\mu \mathrm{m}) ; \tilde{\mathrm{N}}$. peridio (barra=10 $\mu \mathrm{m})$. Tuber tequilanum G. Guevara, G. Bonito \& J. Trappe (Gómez-Reyes 761): O. ascoma (barra=10 mm); P. ascosporas y ascas (barra=10 $\mu \mathrm{m})$; Q. peridio (barra=10 $\mu \mathrm{m})$. 
Hábitat: bosque mesófilo de montaña.

Temporada: julio-septiembre.

Ejemplares examinados: MÉXICO. Michoacán, Uruapan, Estación biológica "Vasco de Quiroga" (La Alberca, Toreo el Alto), 3.VII.2015, Gómez-Reyes 889 (EBUM); loc. cit., 31.VII.2015, Gómez-Reyes 897 (EBUM); loc. cit., 11.IX.2015, Gómez-Reyes 903 (EBUM).

Observaciones: el material revisado coincide con la descripción de Castellano et al. (2012), quienes mencionan que esta especie se caracteriza por poseer micelio color violeta que recubre al ascoma. Otro taxon similar a E. appalachiensis es E. atropurpureus Vittad.; sin embargo, ambas especies se diferencian por la densidad de la ornamentación de las ascosporas, es menos densa en $E$. atropurpureus que en E. appalachiensis. Por otro lado, se observan algunas diferencias en el tamaño de las esporas; Castellano et al. (2012) mencionan las esporas de 7-9 $\mu \mathrm{m}$ de diámetro sin la ornamentación. Sin embargo, los ejemplares del presente trabajo son más grandes, de 8.5-11.5 $\mu \mathrm{m}$ de diámetro, mientras que E. atropurpureus presenta esporas de $10 \mu \mathrm{m}$ de diámetro según Saccardo (1889) y de acuerdo con Paz et al. (2012) son de (8-)9-12(-14) $\mu \mathrm{m}$.

Elaphomyces appalachiensis está citada para los Estados Unidos de América, de los estados de Tennessee, Florida, Iowa, West Virginia, y en México para los estados de Nuevo León y Tamaulipas (Castellano et al., 2012). Se cita por segunda ocasión para el país y por primera vez para el estado de Michoacán; además representa la distribución austral de la especie, la cual se amplía hasta el Sistema Volcánico Transversal.

Genabea cerebriformis (Harkn.) Trappe, Mycotaxon 2(1): 118. 1975. Figs. 1D-F.

= Myrmecocystis candida Harkn., Proc. Calif. Acad. Sci., Ser. 3, Bot. 1: 269. 1899.

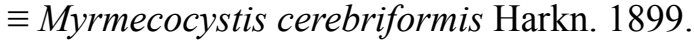

$\equiv$ Genea cerebriformis (Harkn.) Gilkey, University of Calif. Publ. Bot. 6. 1916.

Ascoma 4-7 × 3-4 mm diámetro, subgloboso lobulado; peridio color amarillento (9) a café claro (10), superficie ligeramente verrugosa; gleba irregular con una o varias cámaras; olor y sabor no registrado; peridio 150-350 $\mu \mathrm{m}$, dos capas; epicutis pseudoparenquimatoso 150-250 $\mu \mathrm{m}$ de grosor, color café amarillento en la parte externa, hialina al interior, células globosas, subglobosas e irregulares $12-26 \mu \mathrm{m}$, pared gruesa de $2-4 \mu \mathrm{m}$ de grosor; subcutis $50-80 \mu \mathrm{m}$ de grosor, compuesto de hifas entremezcladas 5-8 $\mu \mathrm{m}$, hialinas, pelos hifales 4-9 $\mu \mathrm{m}$ de ancho, color café, pared gruesa, 1-1.5 $\mu \mathrm{m}$ de grosor, simples, septados; ascas clavadas de (190)200-245 × 17$22 \mu \mathrm{m}$, hialinas, pared delgada, octosporadas; paráfisis septadas, ramificadas con ápice redondeado, 4-6 $\mu \mathrm{m}$ de ancho; ascosporas globosas 27-36 $\mu \mathrm{m}(\mathrm{Q}=1.0-1.03)$, incluyendo la ornamentación, hialinas en agua, densamente ornamentadas, con prominentes espinas 3-5 $\mu \mathrm{m}$ de alto.

Hábito: hipogeo, solitario a gregario bajo Carpinus caroliniana.

Hábitat: bosque mesófilo de montaña.

Temporada: septiembre a octubre.

Ejemplares examinados: MÉXICO. Michoacán, Uruapan, Estación biológica "Vasco de Quiroga" (La Alberca, Toreo el Alto), 2.X.2012, Gómez-Reyes 778 (EBUM), loc. cit., 11.IX.2015, Gómez-Reyes 904 (EBUM).

Observaciones: el género Genabea Tul. \& C. Tul. se separa de Genea, un género afín, por presentar las siguientes características: peridio color amarillento a café, micelio basal ausente, ascosporas generalmente globosas con ornamentación equinulada, ascas elipsoides, clavadas o irregulares con arreglo de ascosporas uniseriado, 
biseriado o irregular e himenio interrumpido por zonas estériles. El género Genea presenta el peridio color café a negro, micelio basal presente, ascosporas elipsoides a subglobosas con ornamentación verrugosa, ascas cilíndricas, arreglo de ascosporas uniseriado e himenio continuo (Trappe, 1975; Smith et al., 2006).

Gilkeya compacta (Harkn.) M.E. Sm. \& Trappe es similar a Genabea cerebriformis; sin embargo, la primera presenta peridio color rojizo además de la ornamentación de las ascosporas que es verrugosa. Asimismo, se separa de las distintas especies de Genea por el peridio color café a negruzco, ascosporas elipsoides a subglobosas con ornamentación verrugosa. Otra especie similar a Genabea cerebriformis es G. sphaerospora Mattir., la cual se distribuye en Europa, con ascosporas más grandes de 35-40 $\mu \mathrm{m}$ de diámetro (Montecchi y Sarasini, 2000), que las de G. cerebriformis que miden 29-34 $\mu \mathrm{m}$ de diámetro (Trappe et al., 2007).

Esta especie se distribuye desde el oeste de los Estados Unidos de América hasta México (Trappe et al., 2009). A pesar de que Trappe et al. (2007; 2009) mencionaron que G. cerebriformis se distribuye en México, no se ha encontrado ninguna descripción con material proveniente del país, por lo que la presente descripción corresponde al primer registro con base en material mexicano.

Genea mexicana Guevara, Göker \& Stielow, Mycol. Progr. 11: 718. 2012. Figs. 1G-I.

Ascoma $10 \times 9 \mathrm{~mm}$ de diámetro, globoso a subgloboso, lobulado, consistencia quebradiza, orificio superior 2-3 mm cubierto con finas vellosidades; peridio color café amarillento (11) a café oscuro (12), cubierto de finas escamas, superficie pubescente, pelos hasta $3 \mathrm{~mm}$ de largo, en la base numerosos rizomorfos color café, reacción ne-

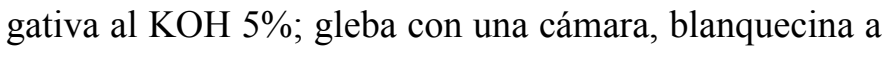
grisácea (2B); epitecio color café claro (10) a café amarillento (11), numerosos pelos hifales; olor y sabor no registrados; peridio 100-350 $\mu \mathrm{m}$ de ancho, dos capas; epicutis pseudoparenquimatosa 50-180 $\mu \mathrm{m}$ de grosor, con células isodiamétricas $15-40 \mu \mathrm{m}$ de diámetro, pared ancha $2 \mu \mathrm{m}$ de grosor, color café en agua y KOH 5\%; pelos hifales 4-9 $\mu \mathrm{m}$ de diámetro, pared gruesa color café rojizo, septados, ramificados, emergen de manera individual o agrupados; subcutis de 50-100 $\mu \mathrm{m}$ de grosor, compuesto por hifas postradas entrelazadas, pared gruesa 5-8 $\mu \mathrm{m}$ de ancho,

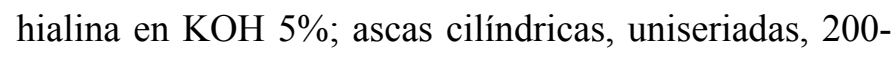
$286 \times 18-23 \mu \mathrm{m}$, pared delgada menor a $1 \mu \mathrm{m}$ de grosor, hialinas en $\mathrm{KOH} 5 \%$, negativo al Melzer, con pedicelo, octosporadas; ascosporas 28-36 × 18-23 $\mu \mathrm{m}$ elipsoides $(\mathrm{Q}=1.4-1.61)$, ornamentación verrugosa (1-3 $\mu \mathrm{m}$ de alto), hialinas en agua, en $\mathrm{KOH}$ 5\% pierden la ornamentación.

Hábito: hipogeo, gregario bajo Quercus L.

Hábitat: bosque de pino-encino con elementos de bosque mesófilo de montaña.

Temporada: julio a septiembre.

Ejemplares examinados: MÉXICO. Michoacán, Morelia, Ichaqueo, 29.VII.2014, Gómez-Reyes 852 (EBUM). Uruapan, Estación biológica "Vasco de Quiroga” (La Alberca, Toreo el Alto), 7.VII.2012, Gómez-Reyes 773 (EBUM).

Observaciones: los ejemplares revisados coinciden con la descripción de Guevara et al. (2012), quienes describieron la especie para el Estado de México y hasta entonces la única localidad de la que se tenía conocimiento, por lo que el presente registro amplía su distribución en el Sistema Volcánico Transversal. Genea mexicana es similar a G. hispidula Berk. ex Tul. \& C. Tul., la cual se diferencia por el tamaño de las ascosporas, G. hispidula presenta ascosporas más grandes, hasta $45 \mu \mathrm{m}$ de largo (Pegler et al., 1993). También es similar con G. arenaria Harkn.; sin embargo, presenta ascosporas más pequeñas, de 20-28 $\mu \mathrm{m}$ de largo (Smith et al., 2006).

Hydnobolites cerebriformis Tul. \& C. Tul., Annls Sci. Nat., Bot., sér. 2, 19: 279. 1843. Figs. 1J-L. 
$=$ H. cerebriformis var. soehneri G. Gross, Z. Mykol. 62(2): 179. 1996.

Ascoma 6-18 × 6-17 $\mathrm{mm}$ diámetro, subgloboso lobulado, irregular; peridio color blanco (2B) a blanco amarillento (3C), superficie lisa, en algunas áreas con hundimientos o pliegues; gleba sólida color blanco (2B) con pequeñas cámaras y venas ramificadas color blanco (2B), algunas llegan a unirse al peridio; olor y sabor no registrados; peridio 70-130 $\mu \mathrm{m}$ de ancho, una sola capa pseudoparenquimatosa con células isodiametricas 15-25 $\mu \mathrm{m}$ de ancho, pared delgada; la superficie del peridio presenta pelos hifales 2-6 $\mu \mathrm{m}$ de ancho, color café, con pared gruesa 1-1.5 $\mu \mathrm{m}$ de ancho, simples y septados; ascas globosas a subglobosas (90-)110-135 × 80-95 $\mu \mathrm{m}$, hialinas, pared delgada, octosporadas; ascosporas globosas 20-30 $\mu \mathrm{m}(\mathrm{Q}=1.0-1.1)$, incluyendo la ornamentación, hialinas en agua, densamente ornamentadas formando 3 a 4 retículos, 2-4 $\mu \mathrm{m}$ de alto.

Hábito: hipogeo, gregario bajo Carpinus caroliniana, Styrax L., Symplocos Jacq., Clethra L. o Quercus.

Hábitat: en cañada con bosque mesófilo de montaña.

Temporada: septiembre.

Ejemplares examinados: MÉXICO. Michoacán, Morelia, Ichaqueo, 16.IX.2014, Gómez-Reyes 871 (EBUM); loc. cit., 20.IX.2014, Gómez-Reyes 876 (EBUM).

Observaciones: Actualmente se reconocen cuatro especies de Hydnobolites: H. californicus E. Fisch., $H$. cerebriformis, $H$. baodingensis E. Wu \& Z. Lan y H. javanicus Höhn., se han registrado para Norteamérica las dos primeras.

Hydnobolites cerebriformis es similar a $H$. californicus; no obstante, se diferencian por el tamaño de las ascosporas más pequeñas (14-18 $\mu \mathrm{m}$ de diámetro) en $H$. californicus (Gilkey, 1954), mientras que H. cerebriformis las presenta de 20.5-24.5 $\mu \mathrm{m}$ de diámetro, incluyendo la ornamentación según Cazares et al. (1992), y de 12-25 $\mu \mathrm{m}$ de diámetro sin incluir la ornamentación con espinas de 2-3 $\mu \mathrm{m}$, de acuerdo con Montecchi y Sarasini (2000).

Hydnobolites cerebriformis se conocía anteriormente del estado de Nuevo León por Cázares et al. (1992); éste registro corresponde a la segunda cita de la especie, ampliando así su área de distribución del norte de México al Sistema Volcánico Transversal.

Tuber pseudoseparans G. Guevara, G. Bonito \& J. Trappe, Ascomycete.org 7(6): 370. 2015. Figs. 1M-Ñ.

Ascoma 6-22 × 4-19 mm de diámetro, subgloboso, consistencia firme; peridio no separable, $\pm 0.5 \mathrm{~mm}$ de grosor, liso pero con depresiones pubescentes, color blanco pálido (2B) a café amarillento (6F); gleba color blanco (2B), en ejemplares jóvenes color café claro (28), apariencia marmolada con venas color blanco; olor y sabor no registrados; peridio 100-200 $\mu \mathrm{m}$ de ancho, dos capas; epicutis pseudoparenquimatosa $50-100 \mu \mathrm{m}$, con células isodiamétricas 7-20 $\mu \mathrm{m}$ de diámetro e hialinas; subcutis 70-100 $\mu \mathrm{m}$, hifas entrelazadas de pared delgada e hialinas 2-5 $\mu \mathrm{m}$; trama sin diferenciación entre la subcutis y las hifas que forman la trama; ascas 1-4(-5) ascosporas 80-120 × 70-90 $\mu \mathrm{m}$, globosas a elipsoides, con pedicelo, hialinas en $\mathrm{KOH}$; ascosporas color café dorado (12), subglobosas a estrechamente elipsoides, 28-65(-70) $\times 24-45$ $\mu \mathrm{m}(\mathrm{Q}=1.12-1.6)$ sin considerar la ornamentación; cantidad de ascosporas variables por asca, 1 ascospora 47.5$65(70) \times 36-38.5 \mu \mathrm{m}(\mathrm{Q}=1.2-1.6) ; 2$ ascosporas $40-51 \times$ $30-40 \mu \mathrm{m}(\mathrm{Q}=1.2-1.5) ; 3$ ascosporas 30-46 $\times 26-35 \mu \mathrm{m}$ $(\mathrm{Q}=1.2-1.35)$; 4 ascosporas $28-37 \times 24-33 \mu \mathrm{m}(\mathrm{Q}=1.12$ $1.34)$; 5 ascosporas $20-34 \times 18-27 \mu \mathrm{m}(\mathrm{Q}=1.1-1.4)$, ornamentación en un retículo alveolado 2-5 $\mu \mathrm{m}$ de alto, 4-8 celdas de lo largo, 3-6 celdas de ancho.

Hábito: hipogeo, gregario bajo Quercus castanea Née y $Q$. obtusata Bonpl. 
Hábitat: bosque de pino-encino.

Temporada: julio a noviembre.

Ejemplares examinados: MÉXICO. Michoacán, Morelia, Ichaqueo, 20.IX.2014, Gómez-Reyes 874 (EBUM). Uruapan, Parque Nacional Barranca del Cupatitzio, 3.X.2009, Gómez-Reyes 210 (EBUM); loc. cit., 4.IX.2010, Gómez-Reyes 375 (EBUM).

Observaciones: Tuber pseudoseparans se distingue del resto de las especies de Tuber P. Micheli ex F.H. Wigg. del grupo Puberulum citadas para México, por el tamaño de las ascosporas, alcanzando hasta $65 \mu \mathrm{m}$ de largo; no obstante, en uno de los ejemplares revisados se encontraron ascosporas hasta de $70 \mu \mathrm{m}$ de largo, las más grandes conocidas para el país. En México se conocía solo para el estado de Jalisco (Guevara et al., 2015b); con el presente reporte se amplía su distribución al estado de Michoacán.

Tuber tequilanum G. Guevara, G. Bonito \& J. Trappe, Ascomycete.org 7(6): 370. 2015. Figs. 1O-Q.

Ascoma $22 \times 19 \mathrm{~mm}$ de diámetro, globoso a subgloboso, consistencia firme; peridio no separable, finamente pubescentes, color blanco (2B), blanquecino (4C), café amarillento (11) al contacto; gleba blanquecina (3C) a color café claro (4D) en ejemplares jóvenes, color café oscuro (31) en ejemplares maduros, apariencia marmolada con venas color blanco (2B); olor y sabor no registrado; peridio 100-200 $\mu \mathrm{m}$ de ancho, dos capas; epicutis pseudoparenquimatosa $100-120 \mu \mathrm{m}$ de ancho, células isodiamétricas de 7-25 $\mu \mathrm{m}$ de diámetro, hialinas, pared ancha; subcutis 60-100 $\mu \mathrm{m}$ de ancho, hifas entrelazadas, hialinas 2-5 $\mu \mathrm{m}$ de ancho, pared delgada; ascas de 1-4(-5) ascosporas, 74-134 × 58-123 $\mu \mathrm{m}$, globosas a elipsoides, paredes de las ascas delgadas cuando joven a gruesas, hialinas en $\mathrm{KOH}$; ascosporas color café amarillento a café dorado (12), subglobosas a estrechamente elipsoides, 24$51 \times 19-36 \mu \mathrm{m}(\mathrm{Q}=1.07-1.7)$ sin ornamentación; ascosporas contenidas, 1 ascospora $33-51 \times 25-36 \mu \mathrm{m}, 2$ as- cosporas 33-43 $\times 25-32 \mu \mathrm{m}, 3$ ascosporas $28-39 \times 23-29$ $\mu \mathrm{m}, 4$ ascosporas $26-38 \times 19-28 \mu \mathrm{m}, 5$ ascosporas $24-36$ $\times 20-26 \mu \mathrm{m}$, ornamentación en retículo alveolado, 2-5 $\mu \mathrm{m}$ de alto, 4-7 celdas de largo, 3-6 celdas de ancho.

Hábito: hipogeo, gregario bajo Quercus castanea, Q. magnoliifolia Née.

Hábitat: bosque de pino-encino y bosque de encino.

Temporada: en septiembre y octubre.

Ejemplares examinados: MÉXICO. Michoacán, Madero, Llano de la Vaquita, 13.IX.2014, Gómez-Reyes 878 (EBUM); Morelia, Atécuaro, Puerto Madroño ejido La Ampliación, 18.X.2011 Gómez-Reyes 761 (EBUM); Ichaqueo, 19.X.2011, Gómez-Reyes 764 (EBUM); loc. cit., 23.VIII.2014, Gómez-Reyes 865 (EBUM), loc. cit., 16.IX.2014, Gómez-Reyes 872 (EBUM).

Observaciones: Los ejemplares revisados coinciden con la descripción de Guevara et al. (2015b). Tuber tequilanum se describió del estado de Jalisco y se distingue por presentar un peridio blanquecino el cual se mancha color café claro al contacto y una gleba café oscura. Tuber tequilanum es similar a T. pseudoseparans; sin embargo, esta última presenta ascosporas de mayor tamaño $(46-65 \times 33-46 \mu \mathrm{m})$ y la gleba es color café claro. Se distingue de T. bonitoi G. Guevara $\&$ Trappe, porque ésta presenta un peridio y ascosporas más anchas que T. tequilanum. El presente registro es la segunda cita para el país y la primera para el estado de Michoacán.

\section{DISCUSIÓN}

El presente trabajo contribuye al conocimiento sobre la taxonomía y distribución de diversas especies de ascomicetos de hábito hipogeo. Con los registros mencionados arriba, el número de hongos hipogeos para el estado de Michoacán se incrementa de 13 a 19 especies, 18 de las cuales se han recolectado en el Sistema Volcánico Transversal. Sin embargo, sigue siendo pobre el conocimiento que se tiene acerca de este grupo y es necesario seguir 
realizando estudios. Debido a su importancia ecológica aportan información relevante, pues forman asociaciones micorrícicas con diversos hospederos de los bosques templados como encinos y pinos entre otras especies, como el caso de Carpinus caroliniana catalogada como Amenazada (A) en la NOM-059-SEMARNAT-2010 (SEMARNAT, 2010), y que se reporta en asociación con Elaphomyces appalachiensis, Genabea cerebriformis e Hydnobolites cerebriformis. Además, los ecosistemas donde se distribuye este grupo de organismos están siendo amenazados por el cambio de uso de suelo para su conversión en huertas de aguacate. Al menos en el estado de Michoacán se ha estimado un incremento en la superficie de producción aguacatera de 75\% de 1980 a 2012 (Chávez-León et al., 2012).

Esta región biogeográfica es de gran importancia biológica, ahí se distribuyen 25 de las 42 especies de ascomicetos hipogeos que se han reportado para el país. El Sistema Volcánico Transversal para algunos grupos de especies representa una barrera o un filtro, mientras que otros lo han aprovechado como un corredor, o han encontrado refugio en alguna de sus numerosas montañas (Rzedowski y Calderón de Rzedowski, 1987; Morón y Ordoñez-Reséndiz, 2015). Por ejemplo, Elaphomyces appalachiensis, de acuerdo con Castellano et al. (2012), se distribuye en el este de los Estados Unidos de América y el noroeste de México (Nuevo León y Tamaulipas) y con el presente registro hasta el occidente del centro del país. Además, esta región alberga un gran número de especies endémicas. Hasta el momento Genea mexicana, Tuber pseudoseparans y T. tequilanum solo se conocen para el Sistema Volcánico Transversal en asociación con diversas especies de encino en altitudes que oscilan entre 2100 y $3100 \mathrm{~m}$ en el caso de G. mexicana (Guevara et al. 2012), y de 1500 y $2300 \mathrm{~m}$ para las dos especies de Tuber. De acuerdo con Guevara et al. (2015b) este último género presenta altos niveles de endemismo continental como resultado de patrones de una limitada dispersión.

\section{CONTRIBUCIÓN DE AUTORES}

VMGR y AMOG realizaron las recolectas. VMGR escribió el manuscrito con la colaboración de GVM, AMOG y
GGG, quienes aportaron en la redacción del trabajo y/o la revisión crítica de su contenido intelectual. Todos los autores contribuyeron a la discusión, revisión y aprobación del manuscrito final.

\section{FINANCIAMIENTO}

Este estudio fue apoyado por el Consejo Nacional de Ciencia y Tecnología (CONACyT).

\section{AGRADECIMIENTOS}

Gómez-Reyes agradece al Consejo Nacional de Ciencia y Tecnología por la Estancia de Posdoctorado Vinculada al Fortalecimiento de la Calidad del Posgrado Nacional convocatoria 2017(2). Guevara Guerrero agradece al Consejo Nacional de Ciencia y Tecnología y al Sistema Nacional de Investigadores por su apoyo económico utilizado en el estudio de los hongos hipogeos de México.

\section{LITERATURA CITADA}

Castellano, M. A., J. M. Trappe, Z. Maser y C. Maser. 1989. Keys to spores of the genera of hypogeous fungi of North temperate forests with special reference to animal mycophagy. Mad River Press. Eureka, USA. 186 pp.

Castellano, M. A., G. Guevara, J. García y J. M. Trappe. 2012. Elaphomyces appalachiensis and E. verruculosus sp. nov. (Ascomycota, Aurotiales, Elaphomycetaceae) from Eastern North America. Revista Mexicana de Micología 35: 17-22.

Cázares, E., J. García, J. Castillo y J. M. Trappe. 1992. Hypogeous fungi from Northern Mexico. Mycologia 84(3): 341-359. DOI: https://doi.org/10.2307/3760186

Chávez-León, G., L. M. Tapia, M. Bravo, J. T. Sáenz, H. J. Muñoz, I. Vidales, A. Larios, J. B. Rentería, F. J. Villaseñor, J. L. Sánchez, J. J. Alcántar y M. Mendoza. 2012. Impacto del cambio del uso del suelo forestal a huertos de aguacate. Libro Técnico No. 13. Instituto Nacional de Investigaciones Forestales, Agrícolas y Pecuarias, Centro de Investigación Pacífico Centro, Campo Experimental Uruapan. Uruapan, México. 76 pp.

Fogel, R. y J. M. Trappe. 1978. Fungus consumption (mycophagy) by small animals. Northwest Science 52: 1-31. 
Garza, F., J. García y J. Castillo. 1985. Macromicetos asociados al bosque de Quercus rysophylla en algunas localidades del centro del Estado de Nuevo León. Revista Mexicana de Micología 1: 423-438.

Gilkey, H. M. 1954. North American Flora, Series II, Part I: Tuberales. The New York Botanical Garden. New York, USA. 36 pp.

Gómez-Reyes, V. M., I. R. Hernández-Salmerón, A. TerrónAlfonso y G. Guevara. 2012. Estudio taxonómico de Elaphomyces spp. (Ascomycota, Eurotiales, Elaphomycetaceae) de Michoacán, México. Revista Mexicana de Micología 36: 57-62.

Gómez-Reyes, V. M., M. Gómez-Peralta, A. Terrón-Alfonso y G. Guevara. 2014. Descripción de Trappea darkeri (Trappeaceae: Hysterangiales) de México. Revista Mexicana de Biodiversidad 85(4): 1265-1268. DOI: https://doi.org/10.7550/rmb.43995

Gómez-Reyes, V. M., M. Gómez-Peralta y G. Guevara. 2017. Primer reporte y distribución de Delastria rosea (Pezizales: Incertae sedis) en México. Acta Botanica Mexicana 119: 139-144. DOI: https://doi.org/10.21829/ abm119.2017.1236

Guevara, G., I. Báez-Alvarado, V. M. Gómez-Reyes y M. A. Castellano. 2015a. Stephanospora michoacanensis (Stephanosporaceae, Agaricales), a novel sequestrate truffle from North America. Revista Mexicana de Micología 41: 71-75.

Guevara, G., G. Bonito y E. Cázares. 2013. Revisión del género Tuber (Tuberaceae: Pezizales) de México. Revista Mexicana de Biodiversidad 84: S39-S49. DOI: https:// doi.org/10.7550/rmb.31981

Guevara, G., G. Bonito, E. Cázares, J. Rodríguez, R. Vigalys y J. M. Trappe. 2008a. Tuber regimontanum, new species of truffle from Mexico. Revista Mexicana de Micología 26: 17-20.

Guevara, G., G. Bonito, E. Cázares-González, R. Healy, R. Vigalys y J. M. Trappe. 2015b. Novel Tuber spp. (Tuberaceae, Pezizales) in the Puberulum Group from Mexico. Ascomycete.org 7(6): 367-37.

Guevara, G., M. A. Castellano y V. M. Gómez-Reyes. 2016. Two new Aroramyces species (Hysterangiaceae, Hyste- rangiales) from México. IMA FUNGUS 7(2): 235-238. DOI: https://doi.org/10.5598/imafungus.2016.07.02.02

Guevara, G., M. A. Castellano, J. García, E. Cázares y J. M. Trappe. 2008b. Hysterangium (Hysterangiales, Hysterangiaceae) from northern Mexico. Revista Mexicana de Micología 28: 95-100.

Guevara, G., B. Stielow, H. Tamm, E. Cázares y M. Göker. 2012. Genea mexicana, sp. nov., and Geopora tolucana sp. nov., new hypogeous Pyronemataceae from Mexico, and the taxonomy of Geopora reevaluated. Mycological Progress 11(3): 711-724. DOI: http://doi.org/10.1007/ s11557-011-0781-y

Healy, R. A., G. Bonito y G. Guevara. 2009. The truffle genus Pachyphloeus in the U.S. and Mexico: phylogenetic analysis and a new species. Mycotaxon 107(1): 61-71. DOI: https://doi.org/10.5248/107.61

Hibbett, D. S., M. Binder, J. F. Bischoff, M. Blackwell, P. F. Cannon, O. E. Eriksson, S. Huhndorf, T. James, P. M. Kirk, R. L. Cking, H. T. Lumbsch, F. O. Lutzoni, P. B. Matheny, D. J. Mclaughlin, M. J. Powell, S. Redhead, C. L. Schoch, J. W. Spatafora, J. A. Stalpers, R. Vilgalys, M. C. Aime, A. Aptroot, R. Bauer, D. Begerow, G. L. Benny, L. A. Castlebury, P. W. Crous, Y. Ch. Dai, W. Gams, D. M. Geiser, G. W. Griffith, C. Gueidan, D. L. Hawksworth, G. Hestmark, K. Hosaka, R. A. Humber, K. D. Hyde, J. E. Ironside, U. Kõljalg, C. P. Kurtzman, K. H. Larsson, R. Lichtwardt, J. Longcore, J. Miadlikowska, A. Miller, J. M. Moncalvo, S. MozleyStandridge, F. Oberwinkler, E. Parmasto, V. Reeb, J. D. Rogers, C. Roux, L. Ryvarden, J. P. Sampaio, A. Schüßler, J. Sugiyama, R. G. Thorn, L. Tibell, W. A. Untereiner, Ch. Walker, Z. Wang, A. Weir, M. Weiss, M. M. White, K. Winka, Y. J. Yao y N. Zhang. 2007. A higher-level phylogenetic classification of the Fungi. Mycological Research 111(5): 509-547. DOI: https://doi.org/10.1016/j.mycres.2007.03.004

Hosford, D. R. y J. M. Trappe. 1980. Taxonomic studies on the genus Rhizopogon, II. Notes and new records of species from Mexico and Caribbean countries. Boletín de la Sociedad Mexicana de Micología 14: 3-15.

Index Fungorum. 2017. An international project to index all formal names in the Fungi kingdom. http://www. indexfungorum.org. (consultado diciembre de 2017). 
Kovács, G. M., J. M. Trappe, A. M. Alsheikh, K. Hansen, R. A. Healy y P. Vági. 2011. Terfezia disappears from the American truffle mycota as two new genera and Mattirolomyces species emerge. Mycologia 103(4): 831840. DOI: https://doi.org/10.3852/10-273

Largent, D., D. Johnson y R. Watling. 1984. How to identify mushrooms to genus III: microscopic features. Mad River Press. Eureka, USA. 148 pp.

Lumholtz, C. 1902. Unknown Mexico Vol. 1. Charles Scribner's Sons. New York, USA. 530 pp.

Maser, C., A. W. Claridge y J. M. Trappe. 2008. Trees, truffles, and beasts, how forest function. Rutgers University Press. New Jersey, USA. 288 pp.

Montecchi, A. y M. Sarasini. 2000. Funghi ipogei d'Europa. Associazione Micologica Bresadola y Fondazione Centro Studi Micologici. Vicenza, Italia. 714 pp.

Moreno, G., M. Lizárraga, M. Esqueda, R. Galán y P. Alvarado. 2012. New records of little-known species of Carbomyces (Carbomycetaceae, Ascomycota). Mycotaxon 120(2): 8998. DOI: https://doi.org/10.5248/120.89

Morón, M. A. y M. M. Ordoñez-Reséndiz, 2015. Phyllophaga (s. str.) villardoi, nueva especie del Eje Neovolcánico Transmexicano (Coleoptera: Melolonthidae: Melolonthinae). Dugesiana 22(1): 39-42.

Motic. 2004. Motic Image Plus 2.0. Motic China group. Co., Ltd. Hong Kong, China. http://www.motic.com

Nuske, S. J., K. Vernes, T. W. May, A. W. Claridge, B. C. Congdon, A. Krockenberger y S. E. Abell. 2017. Redundancy among mammalian fungal dispersers and the importance of declining specialists. Fungal Ecology 27: 1-13. DOI: https://doi.org/10.1016/j. funeco.2017.02.005

Ouanphanivanh, N., Z. Merényi, A. K. Orczán, Z. Bratek, Z. Szigeti y Z. Illyés. 2008. Could orchids indicate truffle habitats? Mycorrhizal association between orchids and truffles. Acta Biologica Szegediensis 52(1): 229-232.

Paz, I., C. Lavoise, L. Barrio, F. Richard y P. A. Moreau. 2012. Propuesta de dos nuevas especies del género Elaphomyces, dos primeras citas para la Península Ibérica y una clave de identificación de las especies del género para Europa. Boletín Micológico FAMCAL 7: 85-104.
Pegler, D. N., B. M. Spooner y T. W. K. Young. 1993. British truffles, a revision of British hypogeous fungi. Royal Botanic Gardens, Kew. Kew, UK. 211 pp.

Piña-Páez, C., R. Garibay-Orijel, G. Guevara-Guerrero y M. A. Castellano. 2017. Descripción y distribución de Hydnotria cerebriformis (Discinaceae: Pezizales) en México. Revista Mexicana de Biodiversidad 88(2): 269274. DOI: https://doi.org/10.1016/j.rmb.2017.03.017

RBGE. 1969. Flora of British Fungi, color identification Chart. 5 ed. Royal Botanic Garden. Edinburgh, UK.

Rzedowski, J. 1978. Vegetación de México. Limusa. México, D.F., México. 432 pp.

Rzedowski, J. y G. Calderón de Rzedowski. 1987. El bosque tropical caducifolio de la región mexicana del Bajío. Trace 12: 12-21.

Saccardo, P. A. 1889. Discomyceteae et Phymatosphaeriaceae. Sylloge Fungorum 8: 1-1143.

SEMARNAT. 2010. Norma Oficial Mexicana. NOM-059SEMARNAT-2010. Protección ambiental-Especies nativas de México de flora y fauna silvestres-Categorías de riesgo y especificaciones para su inclusión, exclusión o cambio-Lista de especies en riesgo. Secretaría del Medio Ambiente y Recursos Naturales. Diario Oficial de la Federación. Cd. Mx., México. 78 pp. http://dof.gob.mx/ nota_detalle.php?codigo $=5173091 \&$ fecha $=30 / 12 / 2010$.

Shefferson, R. P., T. Kull y K. Tali. 2008. Mycorrhizal interactions of orchids colonizing Estonian mine tailings hills. American Journal of Botany 95: 156-164. DOI: https://doi.org/10.3732/ajb.95.2.156

Smith, M. E., J. M. Trappe y D. M. Rizzo. 2006. Genea, Genabea and Gilkeya gen. nov.: ascomata and ectomycorrhiza formation in Quercus Woodland. Mycologia 98(5): 699-716. DOI: https://doi.org/10.1080/15572536.2006.11832642

Splivallo, R., S. Ottonello, A. Mello y P. Karlovsky. 2011. Truffle volatiles: from chemical ecology to aroma biosynthesis. New Phytologist 189(3): 688-699. DOI: https://doi.org/10.1111/j.1469-8137.2010.03523.x

Trappe, J. M. 1975. Generic synonyms in the Tuberales. Mycotaxon 2(1): 109-122.

Trappe, J. M. y E. Cázares. 2006. Tuber guzmanii, a new truffle from Southern México. International Journal of Medicinal 
Mushrooms 8(3): 279-282. DOI: https://doi.org/10.1615/

IntJMedMushr.v8.i3.90

Trappe, M., F. Evans y J. M. Trappe. 2007. Field Guide to North American Truffles: hunting, identifying, and enjoying the world's prized fungi. Ten Speed Press. Berkeley, USA. $136 \mathrm{pp}$.

Trappe, J. M. y G. Guzmán. 1971. Notes on some hypogeous fungi from Mexico. Mycologia 63: 317-332.

Trappe, J. M., G. Guzmán y C. Vázquez-Salinas. 1979. Observaciones sobre la identificación, distribución y usos de los hongos del género Elaphomyces en México. Boletín de la Sociedad Mexicana de Micología 13: 145-150.
Trappe, J. M., R. Molina, D. L. Louma, E. Cázares, D. Pilz, J. E. Smith, M. A. Castellanos, S. L. Miller y M. J. Trappe. 2009. Diversity, Ecology, and Conservation of Truffle Fungi in Forest of the Pacific Northwest. Department of Agriculture, Forest Service, Pacific Northwest Research Station. Portland, USA. 194 pp. 